\title{
COMUNICAÇÃO
}

\section{PRIMEIRO RELATO DE Brachymeria podagrica (fabricius, 1789) (Hymenoptera: Chalcididae) PARASITANDO Squamatoides trivittatus Curran, 1927 (Diptera: Sarcophagidae) NO BRASIL}

\author{
First report on Brachymeria podagrica (fabricius, 1789) (Hymenoptera: Chalcididae) parasitizing \\ Squamatoides trivittatus Curran, 1927 (Diptera: Sarchophagidae) in Brazil
}

\author{
Cláudio Gonçalves Silva ${ }^{1}$, Jean Patrick-Bonani ${ }^{2}$, Carlos Henrique Marchiori ${ }^{3}$, \\ Lucas Castro Torres ${ }^{2}$, Bruno Barbosa Amaral ${ }^{4}$
}

\begin{abstract}
RESUMO
No presente trabalho relata-se a primeira ocorrência no Brasil de Brachymeria podagrica (Fabricius, 1789) (Hymenoptera: Chalcididae) coletada em pupas de Squamatoides trivittatus Curran, 1927 (Diptera: Sarcophagidae) obtidas de rim bovino. A prevalência de parasitismo foi de $4 \%$.
\end{abstract}

Termos para indexação: Rim bovino, mosca, inimigo natural, ocorrência.

\section{ABSTRACT}

This work reports for the first time in Brazil the occurrence of the parasitoid Brachymeria podagrica (Fabricius, 1789) (Hymenoptera: Chalcididae) collected from pupae of Squamatoides trivittatus Curran, 1927 (Diptera: Sarcophagidae) reared from bovine kidney. The parasitism prevalence was $4 \%$.

Index terms: Bovine kidney, fly, natural enemy, occurrence.

(Recebido para publicação em 4 de março de 2004 e aprovado em 26 de agosto de 2004)

Os Sarcophagidae são insetos vivíparos e, raramente, ovovivíparos (LOPES \& LEITE, 1989), sendo conhecidas cerca de 600 espécies dessa família na região Neotropical (SHEWELL, 1987). Esses dípteros são de grande interesse médico-sanitário, sendo sua ocorrência, distribuição e predominância em áreas metropolitanas fatores de grande importância. Sua presença já foi observada em cadáveres humanos e de animais domésticos. Os adultos podem ser atraídos por substâncias em processo de fermentação, decomposição, sangue e ferimentos (MARCHENKO, 1985). A mosca Squamatoides trivittatus Curran, 1927 (Diptera: Sarcophagidae) apresenta um elevado grau de sinantropia, sendo potencial vetor de agentes enteropatógenicos (LINHARES, 1981).

Juntamente com a fauna de moscas, podem ser encontradas várias espécies de himenópteros parasitóides, dentre eles citam-se aquelas pertencentes ao gênero Brachymeria Westood, 1829, que são importantes parasitóides primários de dípteros muscóides, como aqueles pertencentes às famílias Sarcophagidae (BURKS, 1960; GRISSEL \& SCHAUFF, 1990; MARCHIORI, 2001) e Calliphoridae (BISHOP et al., 1996). Algumas espécies desse gênero apresentam importância econômica, pois atacam insetos-pragas (GAULD \& BOLTON, 1988). Brachymeria podagrica (Fabricius, 1789) (Hymenoptera: Chalcididae) ocorre em quase todo o mundo parasitando dípteros sinantrópicos e outros Diptera (DELVARE \& BOUCEK, 1992). Objetivou-se com este trabalho avaliar a ocorrência de parasitóides em pupas de dípteros sinantrópicos.

O estudo foi realizado em uma área de plantio de Eucalyptus sp. no campus da Universidade Federal de Lavras, em Lavras - MG, próximo à área urbana, no período de agosto a dezembro de 2003. Procedeu-se à coleta dos dípteros por meio de armadilhas construídas com lata de coloração preta fosca, medindo cerca de $19 \mathrm{~cm}$ de altura por $9 \mathrm{~cm}$ de diâmetro, com duas aberturas tipo veneziana, localizadas no terço inferior, para permitir a entrada dos insetos.

1. Biólogo - Doutorando do Curso de Entomologia da Universidade Federal de Lavras/UFLA - Caixa Postal 3037 - $37.200-000$ - Lavras, MG Bolsista da CAPES - ziwky@yahoo.com.br

2. Engenheiros Agrônomos - Mestrandos do Departamento de Entomologia/UFLA.

3. Biólogo - Professor Dr. Departamento de Ciências Naturais do Instituto Luterano de Ensino Superior de Itumbiara.

4. Aluno do curso de Agronomia da Universidade Federal de Lavras (Iniciação científica). 
Na parte superior foi aclopado um funil de plástico, com a base voltada para baixo e a parte superior vedada com tecido de malha fina. Utilizou-se rim bovino como isca, depositado no interior das latas sobre uma camada de terra. Dez armadilhas foram penduradas em eucalipto a 1 metro do solo e a 2 metros uma das outras, permanecendo no campo por 15 dias, quando se procedeu à retirada do conteúdo e à reposição do substrato. Para a obtenção dos parasitóides, o conteúdo das armadilhas era colocado em recipientes plásticos contendo uma camada de areia para servir de substrato à pupação e levados para laboratório. As pupas foram retiradas com auxílio de peneira fina e pinça, contadas e depositadas individualmente em cápsulas de gelatina (número 00), até a emergência das moscas e/ou dos parasitóides. Os insetos foram identificados pelo Dr. Carlos Henrique Marchiori, do Departamento de Biologia do Instituto Luterano de Ensino Superior de Itumbiara/GO, sendo parte dos espécimes (VOUCHERS) depositados no Museu Regional da Universidade Federal de Lavras.

Foram obtidas 50 pupas de S. trivittatus, das quais $20 \%$ emergiram adultos, $76 \%$ não emergiram nenhum inseto e de $4 \%$ emergiram dois parasitóides pertencentes à espécie $B$. podagrica. Marchiori et al. (2003), estudando a prevalência de B. podagrica, coletaram durante o período de março de 2001 a abril de 2002188 espécimes desse himenóptero em pupas de: Hemilucilia flavifacies (Engel, 1931) e Chrysomia sp. (Diptera: Calliphoridae); Oxysarcodexia thornax (Walker, 1849), Sarcodexia sp. e Peckia chrysostoma (Wiedenann, 1830) (Diptera: Sarcophagidae) e Ophyra sp. (Diptera: Muscidae). B. podagrica foi coletada também em pupas de Chrysomya albiceps (Wiedemann, 1819) (Diptera: Calliphoridae) durante o período de março a agosto de 2001 em Itumbiara, GO (MARCHIORI et al., 2002). Esse é o primeiro relato de B. podagrica utilizando como hospedeiro S. trivittatus, no Brasil.

\section{REFERÊNCIAS BIBLIOGRÁFICAS}

BISHOP, D. M.; HEATH, A. C. G.; HAACK, N. A. Distribution, prevalence and host associations of Hymenoptera parasitic on Calliphoridae occurring in flystrike in New Zealand. Medical and Veterinary Entomology, Oxford, v. 20, p. 365-370, 1996.

BURKS, B. D. A revision of the genus Brachymeria Westwood in America North of Mexico (Hymenoptera: Chalcididae). Trans. Amer. Ent. Soc., [S.l.], v. 86, p. 238-239, 1960.
DELVARE, G.; BOUCEK, Z. On the new world Chalcididae (Hymenoptera). Mem. Amer. Ent. Inst., [S.l.], v. 53, p. 30-31, 1992.

GAULD, I. D.; BOLTON, B. The Hymenoptera. Oxford: Oxford University, 1988. 33 p.

GRISSEL, E. E.; SCHAUFF, M. E. A handbook of the families of Nearctic Chalcidoidea (Hymenoptera).

Entomol. Soc. Wash., [S.l.], 1990.

LINHARES, A. X. Synanthropy of Calliphoridae and Sarcophagidae (Diptera) in the city of Campinas, São Paulo, Brazil. Revista Brasileira de Entomologia, São Paulo, v. 25, p. 189-215, 1981.

LOPES, H. S.; LEITE, A. C. R. Morphology of the egg of Sarcodexia lambens (Diptera: Sarcophagidae). Memórias do Instituto Oswaldo Cruz, Rio de Janeiro, v. 84, p. 497-500, 1989.

MARCHENKO, M. I. Characteristic of development of the fly Chrysomya albiceps (Wd.) (Diptera: Calliphoridae). Entomologiceskoe Obozrenie, Leningrad, v. 64, p. 79-84, 1985.

MARCHIORI, C. H. Ocorrência de Brachymeria podagrica (Fabricius) (Hymenoptera: Chalcididae) como parasitóide de Peckia chrysostoma (Wiedemann) (Diptera: Sarcophagidae) no Brasil. Entomol. Vect., [S.l.], v. 8, n.4, p. 513-517, 2001.

MARCHIORI, C. H. et al. Ocorrência de Brachymeria podagrica (Fabricius) (Hymenoptera: Chalcididae) como parasitóide de dípteros coletados em diferentes substratos em Itumbiara, Sul de Goiás, Brasil. Arquivo Brasileiro de Medicina Veterinária e Zootecnia, Belo Horizonte, v. 55, n. 2, p.246-248. 2003.

MARCHIORI, C. H.; PEREIRA, L. A.; SILVA FILHO, O. M. Brachymeria podagrica (Fabricius) (Hymenoptera: Chalcididae) as a parasitoid of Chrysomya albiceps (Wiedemann) (Diptera: Calliphoridae): first report in Brazil. Arquivo Brasileiro de Medicina Veterinária e Zootecnia, Belo Horizonte, v. 54, n. 5, p. 555-557, 2002.

SHEWELL, G. E. Sarcophagidae. In: MCALPINE, J. F. et al. Manual of neartic Diptera. Otawa: Agriculture Canada, 1987. p. 1159-1186. 
